\title{
Nota sobre una estela ibérica duplicada (Calaceite, Teruel)
}

\author{
Notice on a duplicate Iberian stela (Calaceite, Teruel)
}

\author{
Ignacio Simón Cornago $(*)$
}

\section{RESUMEN}

Este trabajo llama la atención sobre la duplicación de la conocida estela de Santa Ana (Calaceite), perteneciente al grupo de las llamadas estelas del Bajo Aragón de la 2. ${ }^{\text {a }}$ Edad del Hierro. El error parte del catálogo de estos monumentos que Fernández Fuster editó en 1951. En él interpretó una fotografía parcial de la mencionada estela, publicada en Ars Hispaniae, como otro ejemplar, al que hizo proceder del poblado de San Antonio (Calaceite). Este error ha pervivido en la bibliografía hasta ahora.

\begin{abstract}
The aim of this paper is to point out the duplication of the well-known stele from Santa Ana (Calaceite), which belongs to the so called 'stelae of Lower Aragón'. This mistake starts from the work of Fernández Fuster, who in 1951 published a catalogue of these monuments, considering a photograph in detail of the stele of Santa Ana (published in Ars Hispaniae) as another item of the 'stelae of Lower Aragón', and assigning to it a new provenance (San Antonio, Calaceite). This wrong duplication, making two stelaes from just one, has been kept in later literature.
\end{abstract}

Palabras clave: Península Ibérica; Protohistoria; Iconografía; Grabado en piedra; Lanzas; Jinete.

Key words: Iberian Peninsula; Protohistory; Iconography; Engraved stones; Lances; Rider.

\section{INTRODUCCIÓN}

Uno de los conjuntos de estelas más conocidos y numerosos de la Protohistoria Peninsular

(*) Área de Historia Antigua. Dpto. de Ciencias de la Antigüedad. Universidad de Zaragoza. C/ Pedro Cerbuna 12. 50009 Zaragoza. Correo e.: isimon@unizar.es

Recibido: 9-III-2011; aceptado: 5-V-2011. está constituido por los ejemplares del Bajo Aragón. Se documentan poco más de treinta piezas, la mayoría de las cuales se conservan incompletas o son simples fragmentos. Su iconografía, series de lanzas y jinetes, y el significado de la misma han centrado el interés de la investigación, aunque otros aspectos también han sido objeto de debate: cronología, ubicación y funcionalidad. Este trabajo, sin embargo, se ocupa de un problema mucho más concreto como es la duplicación de uno de los ejemplares de Calaceite.

Fruto de las investigaciones de J. Cabré y P. Bosch Gimpera durante los primeros decenios del siglo XX en el Bajo Aragón, son las tres estelas de Calaceite que editaron en el sexto número del Anuari d'Estudis Catalans (1915-1920): Mas del Rei, San Antonio y Santa Ana. Fernández Fuster (1951), al interpretar en su catálogo de las estelas bajoaragonesas una fotografía del último ejemplar citado como una cuarta estela procedente de Calaceite, duplicó la pieza de Santa Ana (Fig. 1).

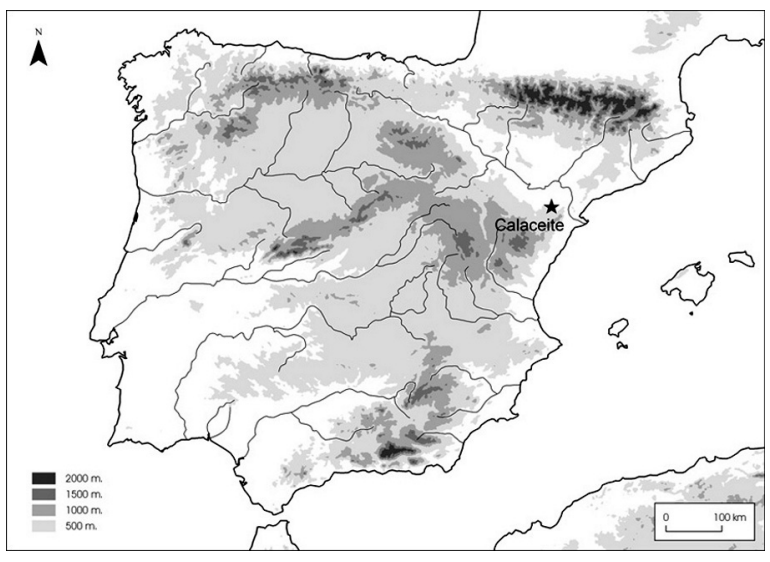

Fig. 1. Localización de Calaceite (Teruel) en la Península Ibérica. 


\section{DESCRIPCIÓN}

1. Mas del Rei (Fernández Fuster 1951: n. ${ }^{\circ}$ 16): solo se conoce la noticia, recogida por J. Cabré (1915-1920: 634), del descubrimiento de una estela, que no pudo ver por haber sido inmediatamente reutilizada como material de construcción en una cabaña (Fernández Fuster 1951: 69-70, n. ${ }^{\circ}$ 16; Marco 1978: 205, II.D.3; Domínguez et al. 1984: n. $\left.{ }^{\circ} 215\right)$.

2. Poblado de San Antonio (Fernández Fuster 1951: n. ${ }^{\circ}$ ) (Fig. 2): fragmento recuperado por P. Bosch Gimpera (1915-1920: 663, fig. 515) entre la runa i terres de la costa Est de la muntan$y a$, entre mig de terrisa, tobes, etc., caiguts de dalt del poblat, del lateral izquierdo de una estela $(35 \times 24 \times 12 \mathrm{~cm})$. El borde está biselado y la superficie frontal dividida por una línea horizontal. Bajo ella se conserva parte de una punta de lanza y sobre ella otras dos lanzas, una de las cuales lleva regatón. Todo ello está grabado en negativo, técnica impropia del conjunto de las estelas del Bajo Aragón, en las que se emplean mayoritariamente líneas incisas para delimitar las siluetas de los elementos representados. Se conserva en el Museu d'Arqueologia de Catalunya. Barcelona (n. ${ }^{\circ}$ inv. 34562).

La importancia cronológica de este hallazgo ha sido señalada por P. Moret (2002: 125-126), puesto que el final del yacimiento, que se sitúa en las postrimerías del siglo III a.C. o inicios del siguiente, proporciona una fecha ante quem previa a la que generalmente se atribuye a estas estelas (siglos II/I a.C.). El carácter fragmentario del hallazgo lo invalida como argumento para defender una localización de este tipo de monumentos en los poblados o en sus proximidades (Bosch 19151920: 641). Por contra, sí está bien atestiguado su reaprovechamiento como material de construcción en los asentamientos. Sucede con varios fragmentos procedentes del yacimiento del Palao (Marco 1976: 90), que curiosamente conservan completa su anchura, muy probablemente porque las estelas fueron cuarteadas horizontalmente para su reutilización, obteniendo así sillares de un módulo más manejable (Cabré 1915-20: 631; Moret 2002: 125; Sanmartí 2007: 241, fig. 4).

3. Camino de Santa Ana (Fernández Fuster 1951: n. ${ }^{\circ}$ 6) (Fig. 3): fue descubierta por un campesino en el transcurso de labores agrícolas en una parcela sita al borde del camino que une Calaceite con la ermita de Santa Ana, próximo a
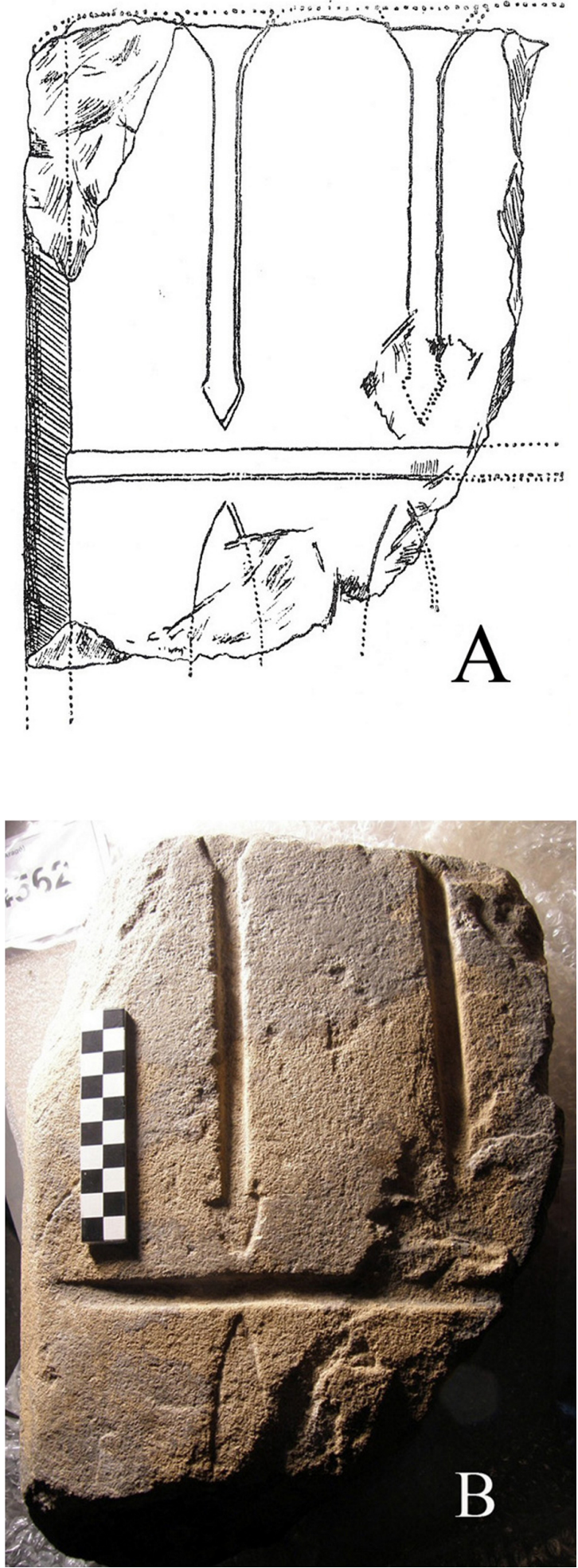

Fig. 2. Estela del poblado de San Antonio, Calaceite (Teruel): A. Dibujo de P. Bosch Gimpera (1915-20: fig. 515); B. Fotografía del autor. 

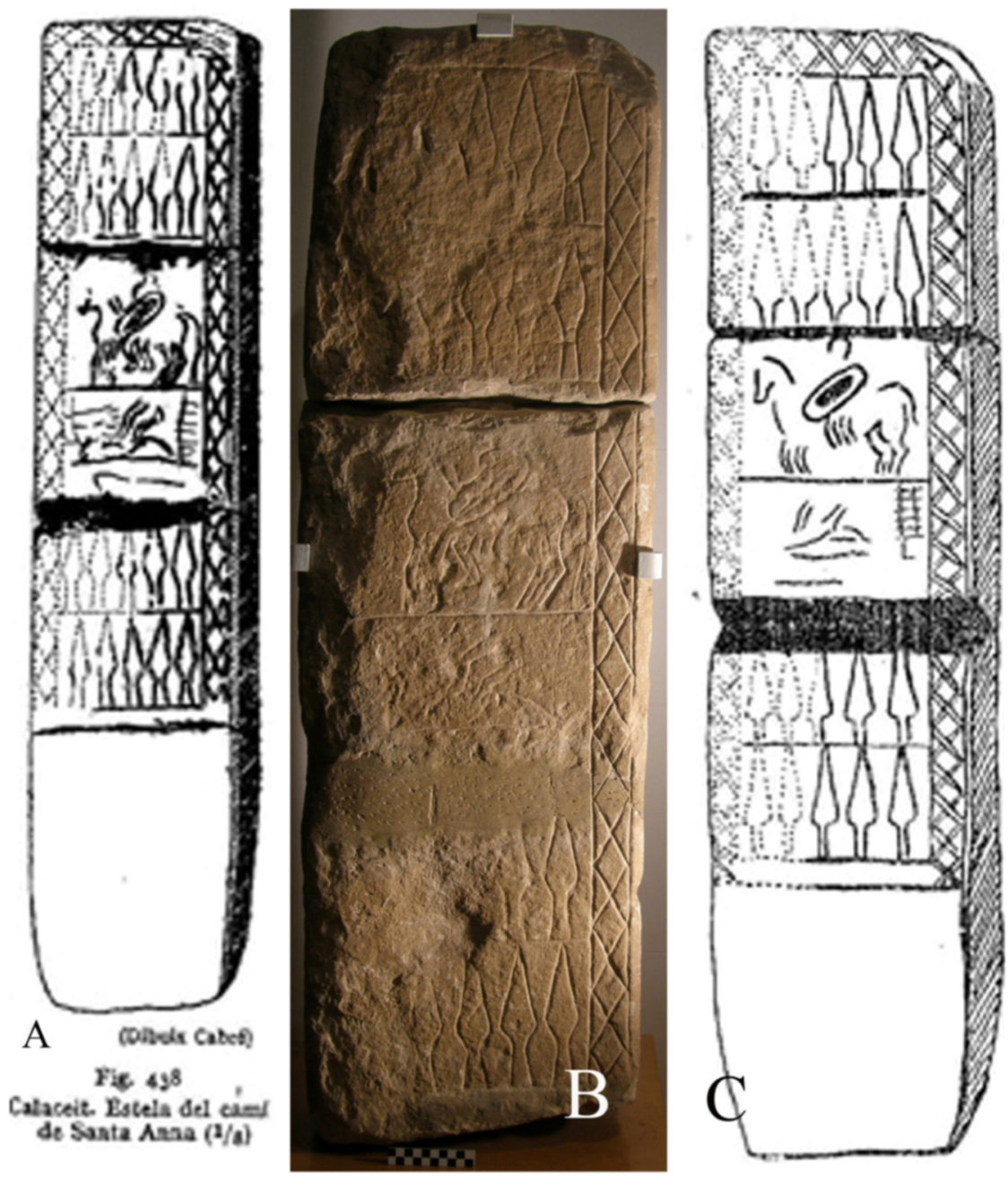

Fig. 3. Estela del camino de Santa Ana, Calaceite (Teruel): A. Dibujo de J. Cabré (1915-1920: fig. 438); B. Fotografía del autor; C. Dibujo de L. Fernández Fuster (1951: Lám. IV).

San Antonio, con otras piezas de dimensiones y forma similares pero sin ornamentar. La pieza fue cuarteada por el labrador y posteriormente recuperada por J. Cabré (1908: 230-233, lám. 3; 1915-1920: 630-631, fig. 438). Actualmente se conserva, en tres partes, en el Museu d'Arqueologia de Catalunya de Barcelona (n. ${ }^{\circ}$ inv. 19880). El campesino informó de que en el mismo lugar halló innombrables blocs de pedra, restes d'urnes, fusaioles, pondus i boletes de fang pintades, restes de cremacions $i$ altres objectes (Cabré 19151920: 630)(1).

(1) Sobre el lugar Moret et al. (2006: 150-151).
La estela mide $131,5 \mathrm{~cm}$ de alto, $39,5 \mathrm{~cm}$ de ancho y $16 \mathrm{~cm}$ de grosor(2). Una orla delimitada por dos líneas incisas y paralelas enmarca la cara frontal en su extremo superior y derecho y, presumiblemente también por el izquierdo, que se conserva muy deteriorado. Dos líneas en zig-zag, configurando rombos, recorren el interior de la orla. El espacio que delimita se divide en seis campos. Los dos inferiores y superiores están ocupados por series de puntas de lanza. El de arriba de los cen-

(2) Cabré (1915-1920: 630) indica una altura de $170 \mathrm{~cm}$; en el dibujo de este autor se señala un fragmento inferior, sin ornar, que actualmente no se conserva. 
trales recoge la imagen de un jinete que porta escudo oval. En el otro -muy erosionado- se aprecian líneas que conforman un diseño geométrico y restos de otra representación de difícil identificación (Bosch 1913-14: 827, fig. 50; Fernández Fuster 1951: 66, n. ${ }^{\circ}$ 6, lám. 4; Marco 1978: 205, II. D.1, fig. 36; Domínguez et al. 1984: n. ${ }^{\circ} 214$ ).

4. Estela duplicada, atribuida a San Antonio (Fernández Fuster 1951: n. ${ }^{\circ}$ 7), aparece por primera vez en el catálogo citado de L. Fernández Fuster (1951). En él compila las estelas publicadas por P. Bosch Gimpera y J. Cabré, a las que añade algunas otras inéditas que Cabré había recogido en volumen del Catálogo Monumental de España dedicado a la provincia de Teruel (inédito). Cataloga las piezas de Mas del Rei y Santa Ana, mientras que adjudica de forma errónea a Palermo (Caspe) la hallada por Bosch Gimpera en San Antonio.
Añade la número 7 de su corpus, que dibuja y localiza en San Antonio de Calaceite (Fig. 4A). Además señala: "depositada en Barcelona. Museo Arqueológico Nacional", dato de interés, pues es la única pieza del catálogo donde se indica la institución en la que se conserva. También ofrece dos referencias bibliográficas de A. García y Be1lido. La primera (García y Bellido 1943: 100 y fig. 125) corresponde a un breve comentario genérico sobre las estelas bajoaragonesas y a una fotografía de la estela con jinete de Palermo (Caspe). La segunda (García y Bellido 1947: fig. 398) se refiere a la fotografía de una estela publicada en Ars Hispaniae con la siguiente nota al pie: "estela de las lanzas, oriunda de Calaceite (Museo de Barcelona)" (Fig. 4B). Es la estela de Santa Ana, de la que sólo se reproducen los campos de la parte superior, con el jinete y las puntas
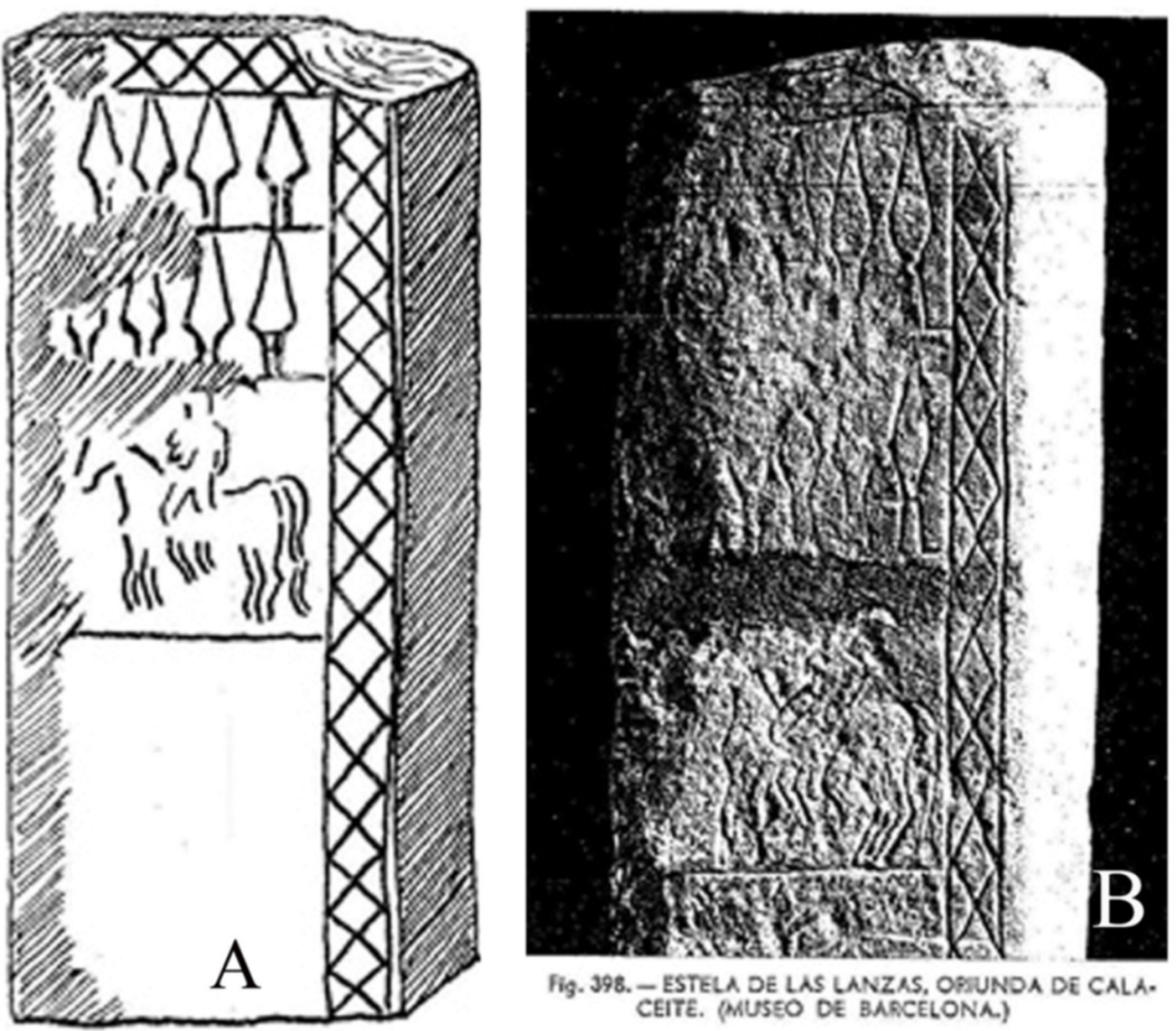

Fig. 4. Estela del camino de Santa Ana, Calaceite (Teruel): A. Fotografía en A. García y Bellido (1947, fig. 398); B. Dibujo que copia la fotografía anterior, L. Fernández Fuster (1951: lám. V). 
de lanza. Es evidente, si se compara la fotografía con el dibujo de Fernández Fuster (1951: lám. V), que éste copia la imagen parcial de la pieza del camino de Santa Ana reproducida en Ars Hispaniae. El autor la describe así: "orla de rombos encuadrando un campo dividido en tres cuarteles, iguales los dos superiores y sensiblemente mayor el inferior. Los dos primeros ostentan lanzas; el inferior un jinete desnudo, al parecer, y tocado de casco o gorro con cimera. Como el de la estela núm. 6 (se refiere a la de Santa Ana) monta a estilo mujeril. Pertenece también a la segunda época". La única diferencia entre los dibujos realizados por Fernández Fuster (1951: lám. IV y V) (Figs. 3C y 4B) de estas supuestas dos piezas es que el escudo que porta el jinete falta en el segundo de ellos, por tratarse de un detalle más difícil de discernir en la imagen de Ars Hispaniae. También añade en la descripción el dato del 'casco o gorro cimerio' que, ni representa en su dibujo, ni se aprecia en la fotografía ni tampoco en el original.

\section{CONCLUSIONES}

Consideramos que Fernández Fuster (1951) interpretó y dibujó como una nueva pieza lo que no era sino una fotografía de la parte superior de la estela de Santa Ana, haciendo proceder a ambas de San Antonio. El error probablemente se debe a que el texto de Ars Hispaniae nada expli- cita sobre la figura en la que aparece la estela, a excepción de la procedencia ("oriunda de Calaceite") y el lugar de conservación (Museo de Barcelona)(3) en el pie de foto. Posiblemente influyó también que en aquellas fechas no se hubiera publicado ninguna reproducción fotográfica del ejemplar de Santa Ana, del que sólo se conocía el dibujo de Cabré. Como resultado del error de la única estela con jinete procedente de Calaceite se hicieron dos, que han persistido en toda la bibliografía posterior (Blázquez 1977: 282; Marco 1978: 205, II.D.2; Domínguez et al. 1984: n. ${ }^{\circ}$ 214; Beltrán 1996: 177-178; Quesada 1994: fig. 1b, 1997: Apéndice V, n. ${ }^{\circ} 71$ ), motivo que, pensamos, justifica esta breve nota.

Otro problema, ya señalado por S. Melguizo (2005: 66), es la incorrecta atribución del lugar de hallazgo de dos de estas estelas. Como ya hemos comentado, Fernández Fuster (1951: 67; también en Marco 1978: 203, II.C.3) catalogó en su trabajo como procedente de Palermo (Caspe) la encontrada por Bosch en San Antonio. Por contra y, casi paradójicamente, la más conocida de las estelas de Palermo (Bosch Gimpera 1915-1920: 665, Fig. 493), en la que aparece representado un jinete, un infante herido y dos series de lanzas, se ha considerado en ocasiones como procedente de San Antonio (Domínguez et al. 1984: lám. XXIII.1; Beltrán 1996: Fig. 176; Quesada 1997: 413-414, Fig. 250, n. ${ }^{\circ}$ 65). La Tabla 1 sintetiza estos errores, surgidos en la bibliografía posterior a la edición de Cabré y Bosch Gimpera.
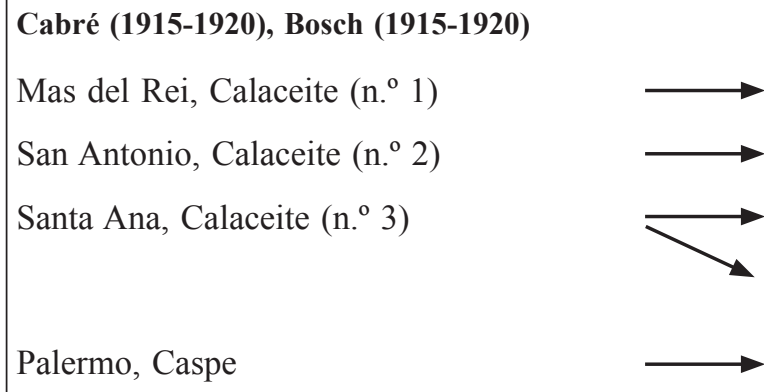

\section{Fernández Fuster (1951)}

Mas del Rei, Calaceite

Palermo, Caspe

San Antonio, Calaceite

Duplicada ("San Antonio, Calaceite") (n. $\left.{ }^{\circ} 4\right)$

Domínguez et al. (1984):

San Antonio, Calaceite

Tab. 1. Cotejo de las atribuciones publicadas sobre las estelas de Calaceite (Teruel).

(3) En el Museo de Barcelona se conservan 8 estelas del Bajo Aragón: la estela del Camino de Santa Ana (n. ${ }^{\circ}$ inv. 19880); el fragmento de San Antonio (n. ${ }^{\circ}$ inv. 34562) recuperado por Bosch; la estela con jinete (n. ${ }^{\circ}$ inv. 19878) y un fragmento con dos puntas de lanza (n. ${ }^{\circ}$ inv. 30655) de Palermo; 2 fragmentos de Mas Pere de la Reina (n. ${ }^{\circ}$ inv. 30654 y 30655; Cabré 191520: Figs. 633,441 y 442$)$ y 2 fragmentos de procedencia insegura (n. ${ }^{\circ}$ inv. 34563 y 34561$)$. 


\section{AGRADECIMIENTOS}

Este artículo se incluye en el proyecto "Los soportes de la epigrafía paleohispánica" (20092010) FFI2009-13292-C03-03. Agradecemos a F. Beltrán y F. Marco sus observaciones sobre este trabajo y a E. Sanmartí su ayuda y colaboración, sin ellas este estudio no hubiera sido posible.

\section{BIBLIOGRAFÍA}

Blázquez, J. M. 1977: “La heroización ecuestre en la Península Ibérica”. En J. M. Blázquez: Imagen y mito: estudio sobre religiones mediterráneas e ibéricas. Ediciones Cristiandad. Madrid: 278-289.

Beltrán, M. 1996: Los íberos en Aragón. Caja de Ahoros de la Inmaculada de Aragón. Zaragoza.

Bosch Gimpera, P. 1913-1914: "Campanya arqueologica de l'Institut d'Estudis Catalans al limit de Catalunya i Aragó (Caseres, Calaceit, Maçalió)”. Anuari del Institut d'Estudis Catalans 5: 819-838.

Bosch Gimpera, P. 1915-1920: "Les investigacions de la cultura ibèrica al Baix Aragò". Anuari del Institut d'Estudis Catalans 6: 541-671.

Cabré, J. 1908: "Hallazgos arqueológicos". Boletín de Historia y Geografía del Bajo Aragón 6: 216-241.

Cabré, J. 1915-1920: "Esteles ibèriques ornamentades del Baix Aragó". Anuari del Institut d'Estudis Catalans 6: 629-641.

Domínguez, A.; Magallón, M. A. y Casado, M. P. 1984: Carta Arqueológica de España. Huesca. Diputación Provincial. Huesca.
Fernández Fuster, L. 1951: "Las estelas ibéricas del Bajo Aragón”. Seminario de arte aragonés 3: 59-76.

García y Bellido, A. 1943: La Dama de Elche y el conjunto de piezas reingresadas en 1941. CSIC. Madrid.

García y Bellido, A. 1947: "El arte de las tribus célticas". Ars Hispaniae. Historia Universal del Arte Hispánico I. Plus Ultra. Madrid: 299-338.

Marco, F. 1976: "Nuevas estelas ibéricas de Alcañiz (Teruel)". Pyrenae 12: 73-90.

Marco, F. 1978: Las estelas decoradas de los conventos Caesaraugustano y Cluniense. Institución Fernando el Católico. Zaragoza.

Melguizo, S. 2005: Íberos en el bajo Regallo. Centro de estudios del Bajo Aragón-Caspe-Institución Fernando el Católico. Caspe.

Moret, P. 2002: "Reflexiones sobre el período ibérico pleno (siglos V a III a. C.) en el Bajo Aragón y zonas vecinas del curso inferior del Ebro". Ilercavonia 3: 111-135.

Moret, P.; Benavente, J. A. y Gorgues, A. 2006: Iberos del Matarraña. Investigaciones arqueológicas en Valdetormo, Calaceite, Cretas y La Fresneda (Teruel). Taller de Arqueología de Alcañiz - Casa de Velázquez. Alcañiz.

Quesada, F. 1994: "Lanzas hincadas, Aristóteles y las estelas del Bajo Aragón". En C. de la Casa (ed.): $V$ Congreso Internacional de estelas funerarias (Soria 1993): 361-369. Soria.

Quesada, F. 1997: El armamento ibérico. Estudio tipológico, geográfico, funcional y simbólico de las armas en la cultura ibérica (siglos VI-I a.C.). Monique Mergoil. Montagnac.

Sanmartí, J. 2007: "El arte de la Iberia septentrional". En L. Abad y J. Soler (eds.): Arte ibérico en la España Mediterránea (Alicante 2005): 239-246. Alicante. 